\title{
AVALIAÇÃO DE DIFERENTES CONFIGURAÇÕES DE IMPELIDORES NA HIDRÓLISE ENZIMÁTICA DO BAGAÇO DE CANA-DE-AÇÚCAR
}

\author{
L.J.CORRÊA ${ }^{1}$, A.C. BADINO ${ }^{1}$, A.J.G. CRUZ ${ }^{1}$ \\ ${ }^{1}$ Universidade Federal de São Carlos, Programa de Pós-Graduação em Engenharia Química - \\ PPGEQ - UFSCar \\ E-mail para contato: ajgcruz@ufscar.br
}

\begin{abstract}
RESUMO: Neste trabalho avaliou-se o desempenho de três configurações de impelidores na hidrólise enzimática do bagaço explodido a vapor: (1) "orelha de elefante" de fluxo descendente e fluxo ascendente; (2) turbina Rushton e "orelha de elefante" de fluxo ascendente; (3) "orelha de elefante" de fluxo descendente e turbina Rushton. Os experimentos foram realizados em reator tipo tanque agitado (3L)

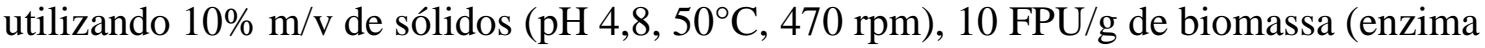
comercial) por $96 \mathrm{~h}$. O consumo de potência foi determinado experimentalmente. A concentração de glicose foi determinada por CLAE e medidas reológicas foram realizadas em reômetro rotacional em frequências de rotação de 10 a $250 \mathrm{rpm}$. Após $24 \mathrm{~h}$, a diminuição da viscosidade se tornou menos acentuada em todas as configurações avaliadas. Na primeira meia hora houve uma acentuada redução no consumo de potência em todas as configurações. As conversões de celulose em glicose obtidas foram de 73,0\%, 66,3\% e 50,5\%, respectivamente para configurações 1, 3 e 2 .
\end{abstract}

\section{INTRODUÇÃO}

A criação do Protocolo de Kyoto em 1997 trouxe exigências mais rígidas aos países desenvolvidos acerca das emissões atmosféricas. O uso de biocombustíveis representa uma das formas mais efetivas de reduzir as emissões de gases do efeito estufa (GEE) associadas ao consumo energético no setor de transporte. O etanol produzido a partir da cana de açúcar chega a reduzir as emissões de GEE em até $90 \%$ quando comparado aos combustíveis fósseis, como a gasolina (Seabra (2008), Farina et al. (2013)).

No Brasil, o etanol produzido a partir da cana de açúcar é o principal combustível substituto da gasolina. É produzido a partir do caldo da cana, sendo denominado etanol de primeira geração (1G). Também pode ser obtido a partir de materiais lignocelulósicos, como o bagaço de cana-de-açúcar, sendo denominado como etanol de segunda geração (E2G) (Corrêa e Cruz, 2013).

No processo de produção do E2G uma das etapas importantes é a hidrólise enzimática. Para que se atinja uma boa eficiência nesta etapa é importante à seleção adequada dos impelidores a serem utilizados e também a obtenção de estimativas de potência, fatores impactantes no custo operacional do processo. 
Sendo assim, este trabalho objetivou avaliar diferentes configurações de impelidores obtendo, experimentalmente, o consumo de potência de cada uma das configurações, a fim de avaliar o melhor conjunto de impelidores na etapa de hidrólise.

\section{MATERIAS E MÉTODOS}

\subsection{Materiais}

Neste trabalho foram empregadas amostras de bagaço de cana-de-açúcar (BCA) explodido a vapor, gentilmente doadas pelo Centro de Tecnologia Canavieira (Piracicaba, SP).

Utilizou-se nos ensaios de hidrólise o complexo enzimático Cellic CTec 2, cedido pela Novozymes Latin America (Araucária, PR).

\subsection{Caracterização do Bagaço}

As amostras de bagaço de cana-de-açúcar explodido a vapor foram caracterizadas de acordo com o procedimento proposto por Gouveia, Nascimento e Rocha (2009). O material foi previamente passado moído em um micro moinho de facas Tipo Willye (modelo SP - 30) a fim de obter-se um material homogêneo, com uma granulometria de $2 \mathrm{~mm}$.

\subsection{Unidade Experimental}

Na Figura 1, apresenta-se a unidade experimental utilizada neste trabalho, a qual consiste em um reator tipo tanque agitado com capacidade útil para 5 litros, um motor de indução trifásico de alto rendimento da marca VOGES (modelo ARB71A6 - E2260) acoplado em um rolamento situado na tampa do reator. A frequência de rotação foi ajustada através de inversor de frequência da marca SIEMENS (modelo Micro Master MM420) A temperatura da reação foi controlada por um banho Ultratermostato da marca Ethik Technology (modelo 521-2D). O consumo de potência foi medido experimentalmente através de um dinamômetro digital da marca LUTRON (modelo FG 6005SD). Acoplado ao motor, tem-se os impelidores utilizados neste estudo e serão descritos no item 2.4
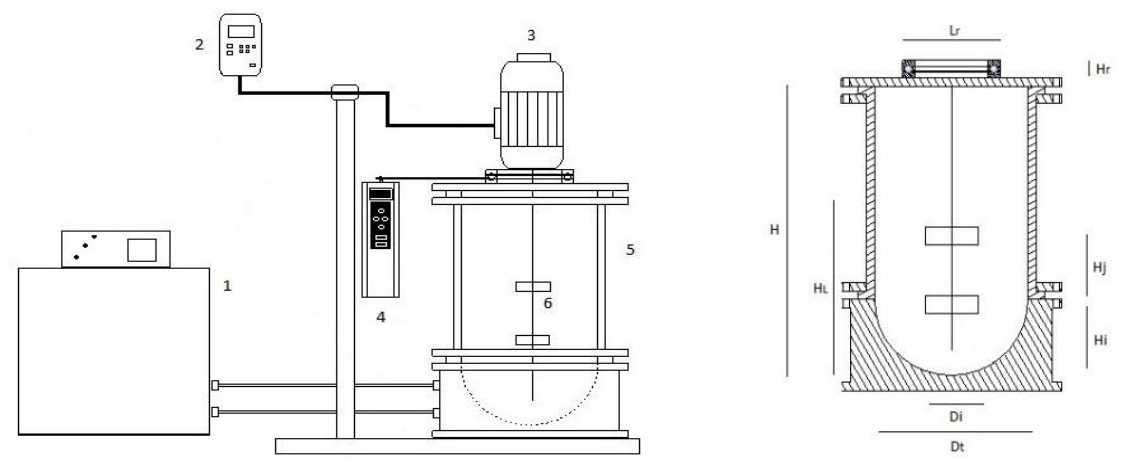

$$
\begin{aligned}
& \mathrm{H}=370 \mathrm{~mm} \\
& \mathrm{H}_{\mathrm{L}}=220 \mathrm{~mm} \\
& \mathrm{Di}_{\mathrm{RT}}=76.5 \mathrm{~mm} \\
& \mathrm{Di}_{\mathrm{EE}}=80 \mathrm{~mm} \\
& \mathrm{Dt}=160 \mathrm{~mm} \\
& \mathrm{Hj}=80 \mathrm{~mm} \\
& \mathrm{Hi}=80 \mathrm{~mm} \\
& \mathrm{Hr}=17 \mathrm{~mm} \\
& \mathrm{Lr}=100 \mathrm{~mm}
\end{aligned}
$$

Figura 1 - Representação esquemática da unidade experimental. (1) Banho Ultra termostato; (2) Inversor de Frequência; (3) Motor; (4) Dinamômetro; (5) Reator; (6) impelidores. 


\subsection{Conjunto de Impelidores}

Foram utilizados três tipos de impelidores neste trabalho a fim de se obter a melhor configuração: impelidores conhecidos como "orelha de elefante" de fluxo ascendente (EEUP) e de fluxo descendente (EEDP) ambos com diâmetro de 0,080m e impelidores tipo turbina Rushton (RT) com diâmetro de 0,076m. Na Figura 2, têm-se os conjuntos de impelidores utilizados.

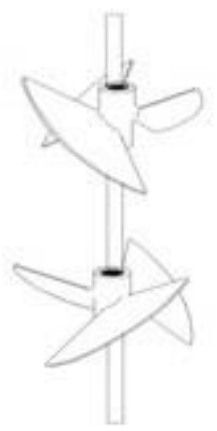

(1)

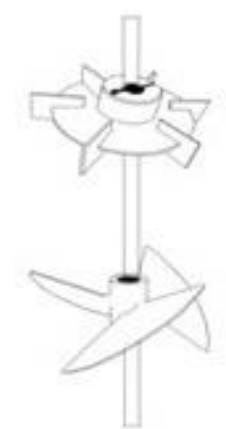

(2)

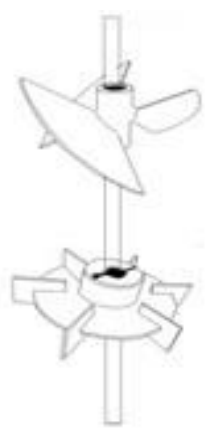

(3)

Figura 2 - Configurações de impelidores empregadas neste trabalho.

$\mathrm{Na}$ configuração (1) tem-se a configuração formada pelos impelidores EEDP EEUP. Na configuração (2) o conjunto é constituído pelos impelidores RT - EEUP. A configuração (3) é formada pelos impelidores EEDP - RT.

\subsection{Tempo de Mistura}

O tempo de mistura $\left(\mathrm{t}_{\mathrm{m}}\right)$ foi determinado pelo método de pulso de temperatura. Consistiu em medir a resposta da temperatura após a adição de uma quantidade de líquido aquecido. Com o auxílio de uma bomba peristáltica foi retirado uma quantidade da solução do reator ( $10 \%$ do volume, equivalente a 303,12 $\pm 1,10 \mathrm{~mL}$ ), aqueceu essa solução em um micro-ondas por 90 segundos. Após isto, a solução foi novamente bombeada para dentro do reator.

Este tempo $\left(\mathrm{t}_{\mathrm{m}}\right)$ foi obtido como o tempo necessário para que o valor da temperatura atingisse $95 \%$ do seu valor final. A medida de temperatura foi feito com um termopar, localizado em uma altura $\mathrm{z}=110 \mathrm{~mm}$ do fundo do reator.

\subsection{Hidrólise Enzimática}

Os experimentos de hidrólise enzimática foram realizados em um reator tipo tanque agitado, descrito no item 2.3, utilizando uma concentração de sólidos de $10 \%$ $(\mathrm{m} / \mathrm{v})$, carga enzimática de $10 \mathrm{FPU} \cdot \mathrm{g}^{-1}$ de biomassa, rotação de $470 \mathrm{rpm}$, solução tampão citrato $(50 \mathrm{mM}, \mathrm{pH} 4,8)$ e com uma temperatura controlada de $50^{\circ} \mathrm{C} \pm 1,0$.

\subsection{Análise de Glicose}

A concentração de glicose foi quantificada por CLAE (cromatógrafo Shimadzu SCL-10A, detector de índice de refração RID10-A, coluna Animex HPX-87H (300 x 7,8mm) da Bio-Rad). Como fase móvel empregou-se solução de ácido sulfúrico 5,0 $\mathrm{mM}$, vazão de $0,6 \mathrm{~mL} \cdot \mathrm{m}^{-1}$ (temperatura do forno: $45^{\circ} \mathrm{C}$ ). 


\subsection{Análise Reológica}

As medidas reológicas foram realizadas nos tempos de 4, 6, 8, 12, 24 36, 48, 60, 72 e 96 horas. Empregou-se volume de $10 \mathrm{~mL}$ do hidrolisado. As medidas foram feitas em reômetro digital (Brookfield, modelo LVDV-III) com spindle (SC4-31) e software Rheocal $2.0^{\circledR}$.

Foram determinadas a viscosidade aparente, a taxa de cisalhamento e a tensão de cisalhamento. A taxa de cisalhamento variou entre 3,4 e $85,0 \mathrm{~s}^{-1}$ para uma faixa de frequência de rotação entre 10 a $250 \mathrm{rpm}$.

\subsection{Consumo de Potência}

A potência consumida $(\mathrm{P})$ durante a hidrólise foi determinada como o produto do torque (T) requerido para a agitação do fluido pela velocidade angular do eixo do impelidor $(\omega)$ conforme a Equação 1.

$$
P=T \cdot \omega
$$

$\mathrm{O}$ torque requerido é calculado pelo produto entre a força $(\mathrm{F})$ medida com o auxílio do dinamômetro e o braço (b), este acoplado ao eixo do motor.

A velocidade angular de rotação do eixo do agitador $(\omega)$ é dada pela Equação 2.

$$
\omega=2 \pi \cdot N
$$

Sabendo-se que para esse sistema o braço equivale a $0,175 \mathrm{~m}$, logo temos a Equação 3.

$$
P=1,1 . F . N
$$

Substituindo-se os valores da Força (F) em "N" e da Frequência de Rotação(N) em "s ${ }^{-1}$ ", os valores da Potência (P) foram calculados em "W".

\section{RESULTADOS}

\subsection{Caracterização do Bagaço}

A composição percentual dos constituintes do bagaço explodido, determinada conforme a metodologia apresentada por Gouveia et al (2009), foi 43,16\% de celulose; $12,44 \%$ de hemicelulose e $34,54 \%$ de lignina e cinzas.

\subsection{Tempo de Mistura}

O tempo de mistura, para as diferentes configurações de impelidores, foi determinado pelo método de pulso de temperatura e os resultados podem ser visualizados nas Figuras 3 (A, B e C) que correspondem às configurações (1), (2) e (3) respectivamente. 

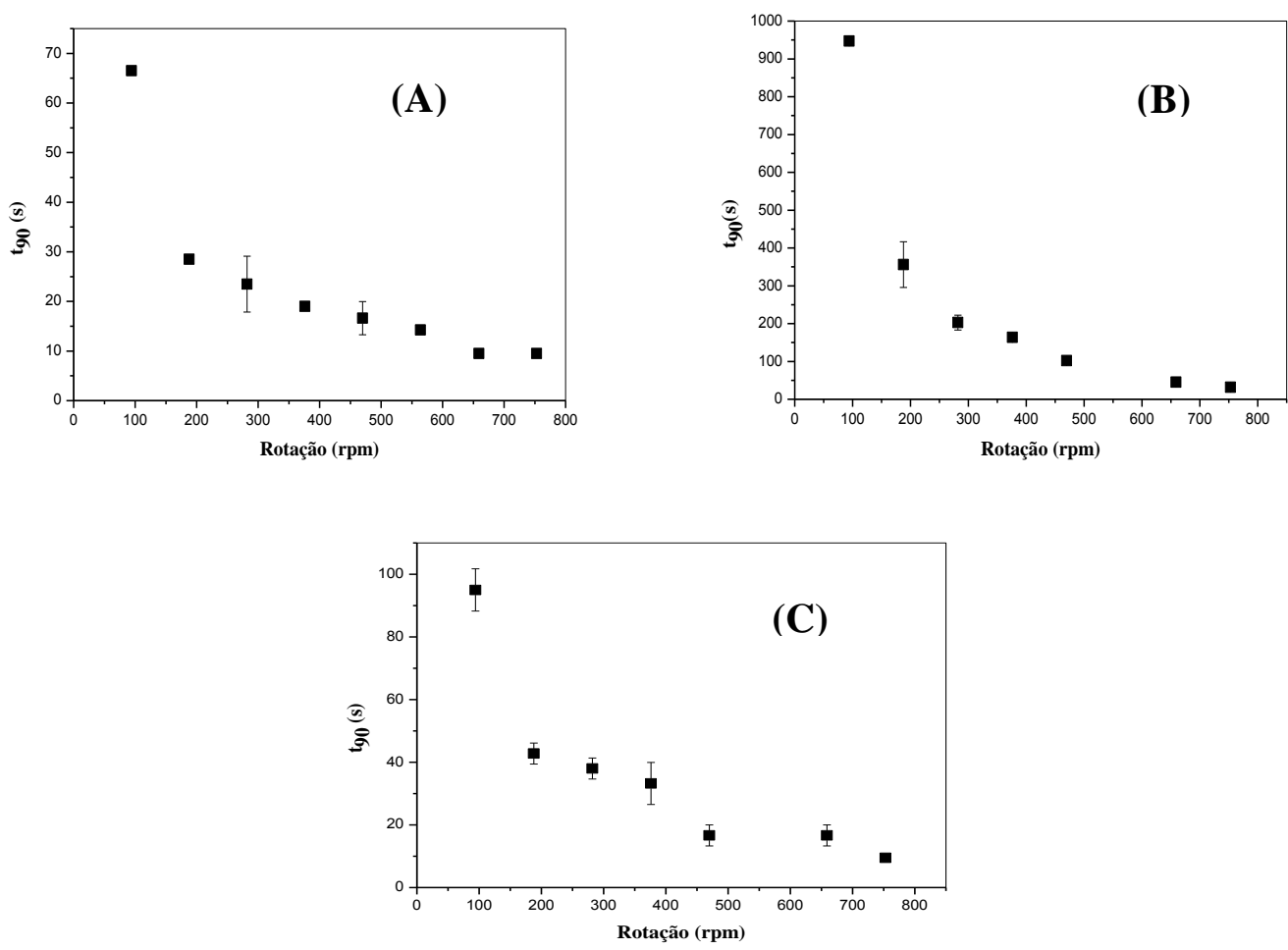

Figura 3 - Tempo de mistura para as configurações: (A) EEDP-EEUP; (B) RT-EEUP;

(C) EEDP-RT.

Baseado nos dados obtidos acima para o tempo de mistura (Figura 4) realizou-se a escolha da rotação a ser utilizada na hidrólise. Em todas as configurações observou-se, visualmente (durante os ensaios) e posteriormente comprovado pelos dados experimentais do tempo de mistura, que na rotação de $470 \mathrm{rpm}$ alcançava-se uma boa mistura do meio reacional. Esta rotação apresentou uma redução no tempo de mistura $(12 \%, 38 \%$ e $50 \%$ para as configurações 1,2 e 3 , respectivamente) quando comparada à rotação de $376 \mathrm{rpm}$.

Para a configuração (1) foi determinado o tempo de mistura na rotação de 564 rpm, obtendo uma redução nesta variável de $14 \%$ em relação a rotação de 470 rpm.

Apesar de rotações mais altas apresentarem um tempo de mistura menor e consequentemente uma melhor mistura, estas rotações consomem mais energia. Dessa forma adotou-se a velocidade de $470 \mathrm{rpm}$ para realização dos experimentos.

\subsection{Análise de Glicose}

A Figura 4 apresenta os valores das concentrações de glicose dos ensaios de hidrólise nas diferentes configurações de impelidores.

Em 96h de reação, as concentrações de glicose foram de 34,67 g.L $\mathrm{L}^{-1}$ para a configuração (1), 23,98 g. $\mathrm{L}^{-1}$ para a configuração (2) e 31,48 g.L $\mathrm{L}^{-1}$ para a configuração (3). A conversão de celulose a glicose neste tempo foi de 73,0\%, 50,5\% e 66,3\% para as configurações (1), (2) e (3), respectivamente. 


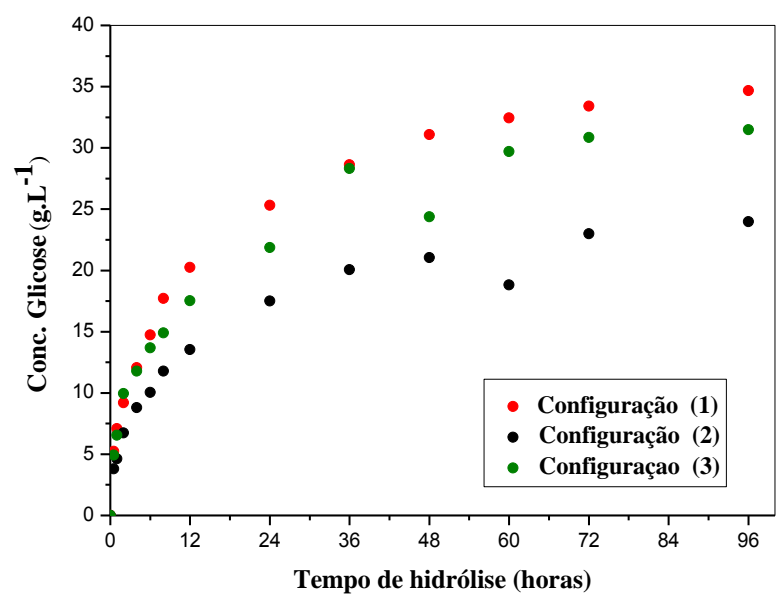

Figura 4 - Concentração de Glicose ao longo da hidrólise.

\subsection{Análise Reológica}

As Figuras 5 (A, B e C), mostram o comportamento da viscosidade aparente em relação ao aumento da taxa de cisalhamento.
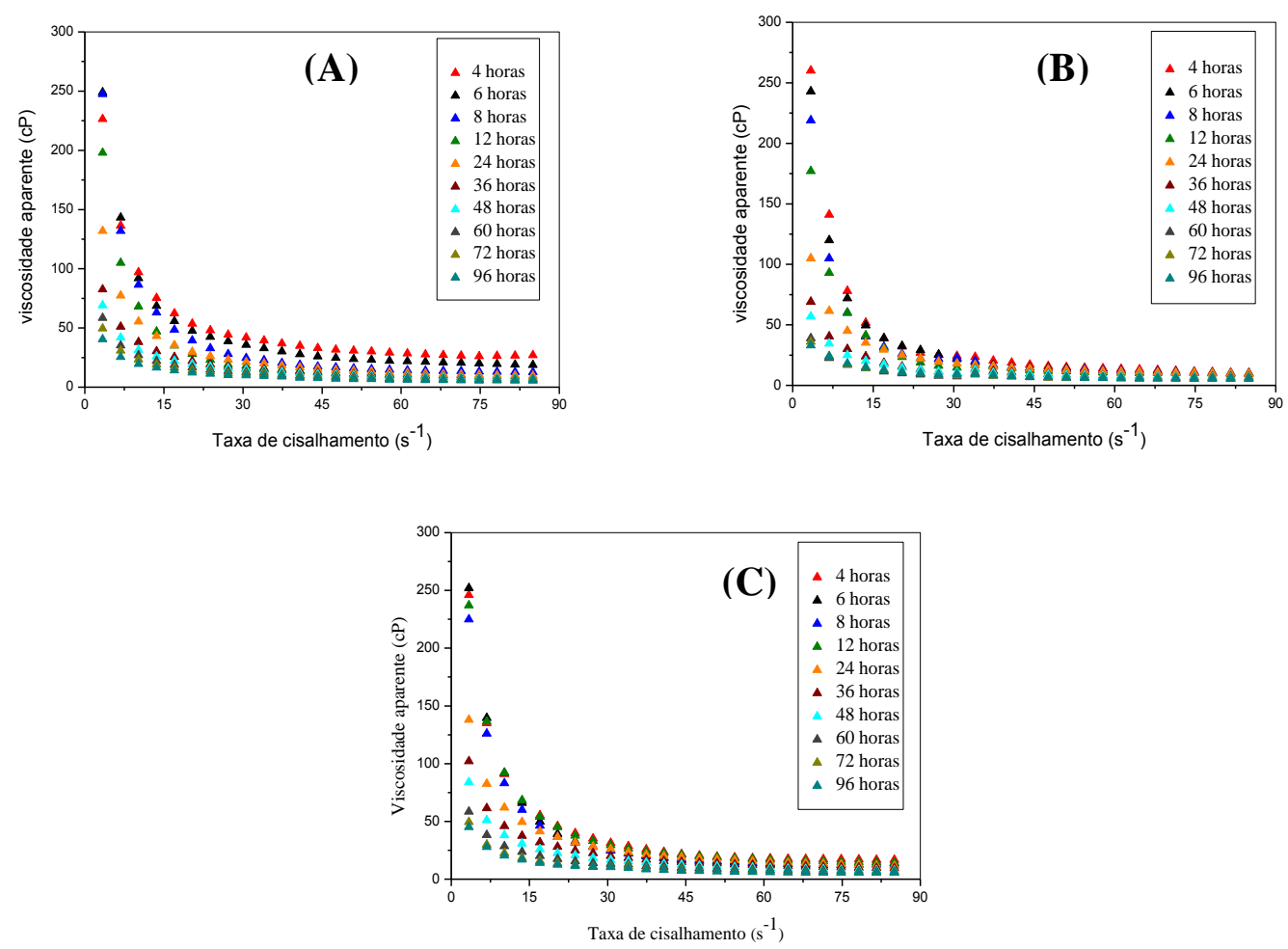

Figura 5 - Dados de viscosidade aparente em função da taxa de cisalhamento nos diferentes tempos de hidrólise. (A) Configuração 1; (B) Configuração 2; (C) Configuração 3.

Com o aumento da taxa de cisalhamento, observa-se uma redução na viscosidade do hidrolisado, uma característica típica de fluido pseudoplástico. Esse 
mesmo comportamento foi observado em estudos do comportamento reológico de hidrolisados provenientes de biomassa (Dasari e Berson (2007), Pereira et. al (2010) e por Corrêa e Cruz (2013)).

Após 12 horas de experimento a redução da viscosidade torna-se menos acentuada em todas as configurações. Essa diferença diminui devido ao avanço da hidrólise ao longo do tempo.

\subsection{Consumo de Potência}

Os perfis do torque, e consequentemente o consumo de energia, das três configurações estudadas reduziram significativamente ao longo das primeiras horas de hidrólise, conforme mostrado na Figura 6.

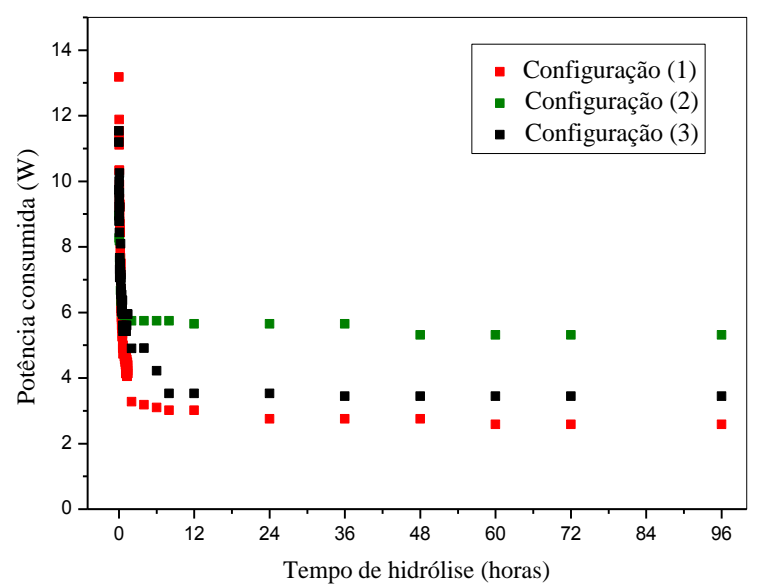

Figura 6 - Consumo de potência durante a hidrólise.

Essa rápida redução no consumo de energia também foi observada por Dasari, Dunaway e Berson (2009) e Palmqvist e Lidén (2012) nos estudos com palha de milho e Arundo, respectivamente.

Esse mesmo comportamento foi observado em todos os conjuntos de impelidores estudados e pode ser explicado devido à degradação das fibras do bagaço ocasionada pela ação das enzimas e com isso modificando a viscosidade do hidrolisado ao longo do experimento.

\section{CONCLUSÃO}

Analisando o conjunto de impelidores, a configuração (1) apresentou os menores tempos de mistura, enquanto a configuração (2) foi a que teve os maiores tempos de homogeneização.

A configuração (1) foi a que apresentou o melhor resultado em termos da conversão da celulose frente aos outros conjuntos. Obteve-se um acréscimo de $44,61 \%$ e $10,15 \%$, comparada com as configurações (2) e (3), respectivamente. 
Os resultados de reologia apresentados mostram que a viscosidade aparente do hidrolisado é dependente da taxa de cisalhamento em todas as configurações, uma vez que a viscosidade decresce com o aumento da taxa de cisalhamento, caracterizando assim este fluido como pseudoplástico.

Apesar do consumo de energia inicial da configuração 1 (13,18 W) tenha sido maior que nas outras configurações, a energia consumida durante toda a hidrólise (96 horas) foi $27,0 \%$ e 7,0 \% menor que a configuração 2 e 3 respectivamente.

\section{AGRADECIMENTOS}

Ao programa Bioen-FAPESP e à CAPES pelo auxílio financeiro.

\section{REFERÊNCIAS}

CORRÊA, L.J.; CRUZ, A.J.G. Estudo do comportamento reológico de amostras de bagaço de cana-de-açúcar durante ensaio de hidrólise enzimática. XIX SINAFERM - X SHEB, Foz do Iguaçu, 2013.

DASARI, R.K.,BERSON, R.E. The effect of particle size on hydrolysis reaction rates and Rheological Properties in cellulosic slurries. Appl. Biochem. and Biotech., p. 288-299, 2007.

DASARI R.K, DUNAWAY K, BERSON R.E. A Scraped Surface Bioreactor for Enzymatic Saccharification of Pretreated Corn Stover Slurries. Energy Fuel, p. 492-497, 2009.

FARINA, E.; RODRIGUES, L.; SOUSA, E.L. A política de Petróleo e a Indústria de etanol no Brasil. Revista Interesse Nacional, 2013.

GOUVEIA, E. R.; NASCIMENTO, R. T.; ROCHA, G. J. M. Validação de metodologia para a caracterização química de bagaço de cana-de-açúcar. Química Nova, v.32, p.1500-1503, 2009.

PEREIRA, L.T.C., BON, E.P.S, TEIXEIRA, R.S.S., PEREIRA, L.T.C, FREITAS, S.P. Sugarcane bagasse enzymatic hydrolysis: rheological as criteria for impeller selection. J. Ind. Microbiol. Biotechnol., p. 901-907, 2011.

SEABRA, J.E.A. Avaliação técnico-econômica de opções para o aproveitamento integral da biomassa de cana no Brasil. Campinas, Faculdade de Engenharia Mecânica, Unicamp. Tese de Doutorado, 273p, 2008. 